INL/EXT-15-37533 Rev. 0

December 2015

\title{
Assessment of MARMOT Grain Growth Model
}

\author{
B. Fromm \\ Y. Zhang \\ D. Schwen \\ D. Brown \\ R. Pokharel
}

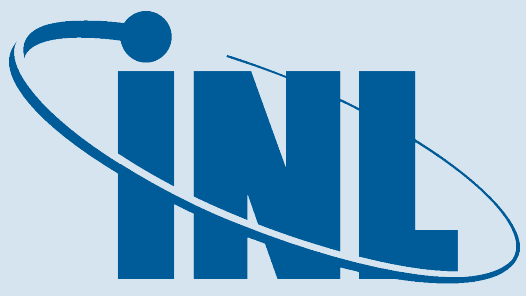

Idaho National Laboratory 


\section{NOTICE}

This information was prepared as an account of work sponsored by an agency of the U.S. Government. Neither the U.S. Government nor any agency thereof, nor any of their employees, makes any warranty, express or implied, or assumes any legal liability or responsibility for any third party's use, or the results of such use, of any information, apparatus, product, or process disclosed herein, or represents that its use by such third party would not infringe privately owned rights. The views expressed herein are not necessarily those of the U.S. Nuclear Regulatory Commission. 


\title{
Assessment of MARMOT Grain Growth Model
}

\author{
B. Fromm \\ Y. Zhang \\ D. Schwen \\ D. Brown \\ R. Pokharel" \\ December 2015 \\ Idaho National Laboratory \\ Fuel Modeling and Simulation Department \\ Idaho Falls, Idaho 83415 \\ *Los Alamos National Laboratory, Los Alamos, NM \\ Prepared for the \\ U.S. Department of Energy \\ Office of Nuclear Energy \\ Under U.S. Department of Energy-Idaho Operations Office \\ Contract DE-AC07-99ID13727
}




\section{ABSTRACT}

This report assesses the MARMOT grain growth model by comparing modeling predictions with experimental results from thermal annealing. The purpose here is threefold: (1) to demonstrate the validation approach of using thermal annealing experiments with non-destructive characterization, (2) to test the reconstruction capability and computation efficiency in MOOSE, and (3) to validate the grain growth model and the associated parameters that are implemented in MARMOT for $\mathrm{UO}_{2}$. To assure a rigorous comparison, the 2D and 3D initial experimental microstructures of $\mathrm{UO}_{2}$ samples were characterized using non-destructive Synchrotron x-ray. The same samples were then annealed at $2273 \mathrm{~K}$ for grain growth, and their initial microstructures were used as initial conditions for simulated annealing at the same temperature using MARMOT. After annealing, the final experimental microstructures were characterized again to compare with the results from simulations. So far, comparison between modeling and experiments has been done for $2 \mathrm{D}$ microstructures, and 3D comparison is underway. The preliminary results demonstrated the usefulness of the non-destructive characterization method for MARMOT grain growth model validation. A detailed analysis of the $3 \mathrm{D}$ microstructures is in progress to fully validate the current model in MARMOT. 


\section{CONTENTS}

FIGURES

1 Introduction $\quad 1$

2 MARMOT grain growth model 2

2.1 Theory of grain boundary migration . . . . . . . . . . . . . . . 2

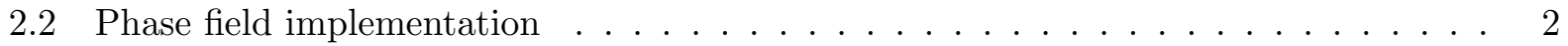

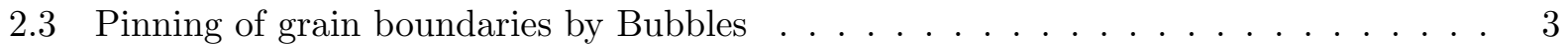

2.4 MOOSE GrainTracker . . . . . . . . . . . . . . . . . . . . . 3

3 Comparison With Experiment 5

3.1 Reconstruction of experimental microstructure . . . . . . . . . . . . . 5

3.2 Comparison of grain growth results from modeling and experiments $\ldots \ldots \ldots$

4 Summary $\quad 9$

5 References $\quad 10$ 


\section{FIGURES}

1 Example of grain remapping with GrainTracker algorithm. Bounding spheres are used to determine when two grains assigned to a common order parameter intersect. Once an intersection is determined, one of the grains is dynamically remapped to a different order parameter. In this example, grain 10 is reassigned from order parameter 2 to order parameter $6 \ldots \ldots \ldots \ldots \ldots$

2 Example of MARMOT input file showing the header information and the first 5 rows of data . . . . . . . . . . . . . . . . . . . . . . . . 6 6

3 Phase field simulation results of UO2-524 microstructure; (a) reconstructed dataset containing 1620 grains at the beginning of simulation; (b) evolved microstructure after 200 minutes of simulated annealing at 2273K. The simulation took 85 time steps to reduce the number of grains to $600 \ldots \ldots \ldots \ldots \ldots$

4 2D grain growth results for UO2-S7 dataset; (a) initial microstructure with 2091 grains; (b) evolved microstructure contains 502 grains after 156 minutes of simulated annealing at $2273 \mathrm{~K}$; (c) experimental dataset contains 257 grains after 156 minutes

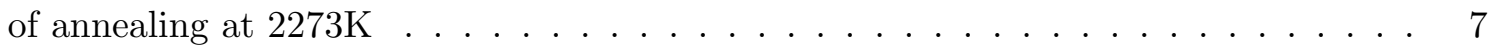

$5 \quad$ Number of grains in a 2D slice as a function of annleaing time at $2273 \mathrm{~K} \ldots \ldots$. . . 8 


\section{Introduction}

Under the Department of Energy (DOE) Nuclear Energy Advanced Modeling and Simulations (NEAMS) program, a microstructure based fuel performance modeling toolkit, the MOOSE BISON - MARMOT (MBM) toolkit, is under development [1]. In the MBM approach, the microstructure of fuels (including both fuel and cladding) is represented by a list of state variables including grain size, porosity, fracture and so on. The state variables evolve with time during fuel operation, representing the microstructure evolution handled by the MARMOT code [2]. The fuel properties will then be determined using the current microstructure in MARMOT, and fed into the BISON code [3] for engineering scale fuel performance modeling. Both MARMOT and BISON are applications developed based on the MOOSE framework.

Grain size is one of the most crucial state variables that affect many fuel properties [4]. In a polycrystalline solid such as a $\mathrm{UO}_{2}$ fuel, grain size determines the density of grain boundaries (GBs) in a unit volume. In $\mathrm{UO}_{2}$, GBs can serve as efficient diffusion paths for fission gases, preferential bubble formation sites, and fracture paths, thus affecting fission gas release and the mechanical strength. GBs themselves and the associated gas bubbles also have significant effects on the thermal conductivity of fuels, particularly at high burn-ups. Therefore, it is important to accurately predict the evolution of grain size during fuel operation. Over the past few years, a grain growth model has been developed in MARMOT with various driving forces including GB energy, thermal gradient, and strain energy. The model needs to be validated before being used in fuel performance modeling.

The grain size in a polycrystalline solid may change via GB migration driven by various driving forces [5]. This phenomenon is called grain growth when the grain size increases. The migration of GBs is driven by the reduction of the overall free energy of the system. This reduction can be due to various sources of energy, including reduction in GB energy, elastic energy, or defect energy. In $\mathrm{UO}_{2}$ fuels, two relevant driving forces have been identified and they are the intrinsic GB energy and the thermal gradient. During operation, the temperature drops from about $1800 \mathrm{~K}$ at the center to about $800 \mathrm{~K}$ at the rim, giving a high thermal gradient over a short distance of about $5 \mathrm{~mm}$. Along with the GB energy, such a high thermal gradient may induce GB migration and thus grain growth. To evaluate the effect of thermal gradient on grain size, MARMOT grain growth simulations were performed with both the GB energy and the thermal gradient driving forces [6]. It was concluded that in spite of the high thermal gradient, the GB energy is still the dominant factor for grain growth in $\mathrm{UO}_{2}$ fuels during operation. Therefore, our validation will focus on grain growth under the intrinsic GB energy driving force.

To validate the grain growth model, thermal annealing experiments of two $\mathrm{UO}_{2}$ samples at high temperatures were conducted by our collaborators at Los Alamos National Laboratory (LANL). During thermal annealing the only driving force for grain growth comes from the intrinsic GB energy. The purpose here is threefold: (1) to demonstrate the validation approach of using thermal annealing experiments with non-destructive characterization, (2) to test the reconstruction capability and computation efficiency in MOOSE, and (3) to validate the grain growth model and the associated parameters that are implemented in MARMOT for $\mathrm{UO}_{2}$. The grain structures of the samples before and after annealing were characterized using non-destructive synchrotron x-ray. The initial grain structures were reconstructed using the MOOSE EBSDReader as the initial conditions for MARMOT grain growth simulations. Such a rigorous comparison allows for a quantitative validation of the grain growth model. In the following, we will introduce the MARMOT grain growth model in Section 2, and the comparison with experiments in Section 3. The conclusion is drawn in Section 4. 


\section{MARMOT grain growth model}

In this Section, the grain growth model in the MARMOT code is briefly introduced. In addition to the GB energy driving force, the thermal gradient driving force is also included, although the latter was not included in the current validation effort for its negligible effect. The grain growth model in MARMOT also includes the effect of pinning by second phase particles such as gas bubbles and intermetallic phases.

\subsection{Theory of grain boundary migration}

Grain boundaries migrate under various driving forces to minimize the overall free energy of the material system. At the low driving force regime, the velocity, v, at which GBs migrate is proportional to the driving fore (as shown in Eq.1), with the proportionality defined as the mobility [5]. where $\mathrm{M}$ is the GB mobility and $\mathrm{P}$ is the driving force. The driving force $\mathrm{P}$ takes the form of an energy per unit volume.

$$
v=M P
$$

For GB energy driving grain growth,

$$
P=\frac{\gamma_{G B}}{R}
$$

Here, $\gamma_{G B}$ and $\mathrm{R}$ are the GB energy and the grain size (curvature), respectively.

While under thermal gradient driving force, we have

$$
P=\frac{\triangle S w_{G B}}{\Omega_{a}} \nabla T
$$

In Eq.3 $\triangle S$ is the excess entropy of GBs in reference to perfect crystal in the same material. $\mathrm{w}_{G B}$ and $\Omega_{a}$ are the corresponding GB width and molar volume. Please note that the above equations are defined on individual GBs and the parameters are GB-specific when GBs are anisotropic. For isotropic GBs, the same set of parameters are shared by all GBs.

\subsection{Phase field implementation}

MARMOT is a phase field based code. To model grain growth in phase field, we employed the quantitative grain growth model from Moelans et al. [7]. In phase field, each grain in the system is represented by an order parameter $\phi_{i}$. The evolution of the order parameters is governed by the Allen-Cahn equation by:

$$
\frac{\partial \phi_{i}}{\partial t}=-L\left(\frac{\partial F}{\partial \phi_{i}}+A \nabla T \cdot \nabla \phi_{i}\right)
$$

Here, $F$ is the total free energy of the system with the contribution from GB energies. The second term in the right hand side of Eq.4 accounts for the thermal gradient driving force. The magnitude of the thermal gradient driving force is given by the parameter A by

$$
A=-\frac{3}{4} \frac{\triangle S w_{G B} w_{i n t}}{\Omega_{a}}
$$

In Eq. $5 \mathrm{w}_{\text {int }}$ is the GB width in the phase field model and different from the physical GB width $\mathrm{w}_{G B}$. 
To allow for quantitative grain growth simulations, we used the GB energy calculated by molecular dynamic simulations, with $\gamma_{G B}=1.58 \mathrm{~J} / \mathrm{m}^{2}$ [8] for average GBs. The GB mobility is taken from previous experimental study, with $\mathrm{M}=9.21 \times 10^{-9} \mathrm{e}^{-\frac{2.77 e v}{K_{B} T}} \mathrm{~m}^{4} / \mathrm{Js}[9]$. Here $\mathrm{K}_{B}$ is the Boltzmann constant and $\mathrm{T}$ is temperature. The GB width is taken as $\mathrm{w}_{G B}=0.5 \mathrm{~nm}$. When thermal gradient driving force is considered, we used an $\nabla S=8.0 \mathrm{~kJ} /(\mathrm{K} \cdot \mathrm{mol})$, and an $\Omega_{a}=7.11 \times 10^{-6} \mathrm{~m}^{3} / \mathrm{mol}$. Please note that here we assume isotropic GB properties.

\subsection{Pinning of grain boundaries by Bubbles}

The motion of GBs can be pinned by the presence of impurities and second phase particles [5]. Impurities that segregate at GBs can reduce the intrinsic mobilities of GBs. Such an effect has not be considered in MARMOT yet. The presence of second phase particles such as gas bubbles exerts a pinning force on GBs, partially or fully canceling the driving forces for GB motion. For instance, porosity exists in both fresh and irradiated fuels. In the latter the porosity evolves due to the evolution of fission gas bubbles. Therefore, the effect of bubble pinning needs to be considered in the grain growth model in MARMOT. For this purpose, We developed an analytical pinning model that is a function of the GB fractional coverage, the percentage of the GB covered by gas bubbles [10]. The model also considers the impact of the bubble size distribution, in terms of the mean and standard deviation of the bubble radius. A significant finding from the model is that the mean value of the resistive pressure decreases with increasing standard deviation of the bubble radius. More information on this pinning model can be reached in Tonks et al.
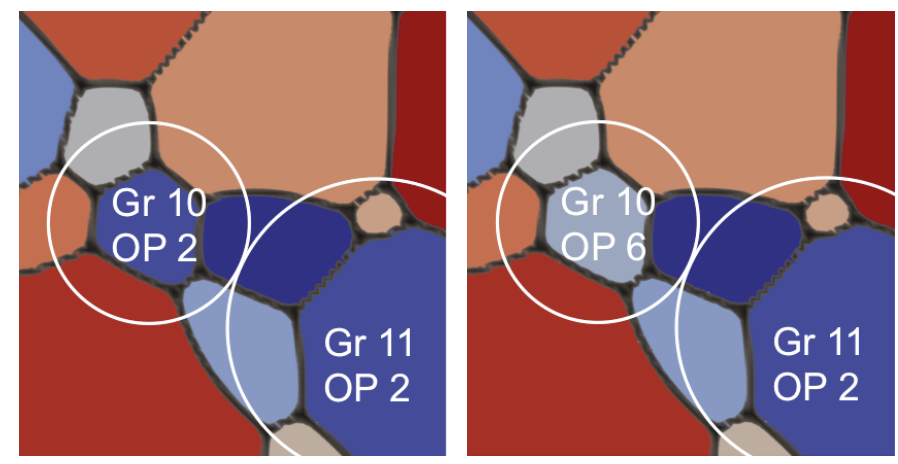

Figure 1: Example of grain remapping with GrainTracker algorithm. Bounding spheres are used to determine when two grains assigned to a common order parameter intersect. Once an intersection is determined, one of the grains is dynamically remapped to a different order parameter. In this example, grain 10 is reassigned from order parameter 2 to order parameter 6

\subsection{MOOSE GrainTracker}

In traditional phase field grain growth models, each grain requires a unique order parameter. For simulations involving thousands of grains, at each material point the evolution equations for all order parameters need to be solved and the corresponding information needs to be stored. This will tremendously increase the memory requirement and reduce the computation efficiency. This problem is resolved by using the GrainTracker tool in MOOSE, taking the advantage that for an individual grain, only the order parameters of its neighboring grains are relevant and need to be explicitly represented. GrainTracker is a modular, dynamic, and scalable reassignment algorithm that groups order parameters together to substantially reduce memory usage. The GrainTracker 
script combines two algorithms: a flood algorithm that identifies the individual grains and a grain tracking algorithm that tracks movement, detects contact, and remaps grains when necessary. To exclude neighboring grains with common order parameters, a bounding sphere is defined for each grain. If the bounding spheres of two grains intersect, the grains will be remapped with one of the grains remapped to a different order parameter. In Fig.1 an example of such remapping process is shown. The bounding spheres of grain 10 and 11, both with order parameter 2, touch each other and trigger the remapping process, which remapped grain 10 to order parameter 2. The grain tracker algorithm has been documented in details in Gaston et al. [11]. and it was used in all MARMOT grain growth simulations included in this report, 


\section{Comparison With Experiment}

In this Section the reconstruction of experimental microstructure and the comparison between MARMOT grain growth simulations and the results from thermal annealing experiments are presented. So far, comparison was made for $2 \mathrm{D}$ simulations only. 3D comparison will be made after reconstructing the experimental microstructures.

\subsection{Reconstruction of experimental microstructure}

The thermal annealing experiments of $\mathrm{UO}_{2}$ samples were conducted at LANL. A high temperature of $2273 \mathrm{~K}$ was used to have substantial grain growth in a reasonable time, e.g., a few hours. The grain structures of the samples were characterized using non-destructive synchrotron x-ray to allow for the using of the same sample for both characterization and the following thermal annealing. Two $\mathrm{UO}_{2}$ samples were used in the experiments, UO2-254 and UO2-S7. The UO2-254 sample is non-stoichiometric, annealed at $2273 \mathrm{~K}$ for 200 minutes. As the effect of off-stoichiometry on GB mobility has not been included in the MARMOT grain growth model yet, the UO2-254 sample is not used for the validation. Only its initial structure was reconstructed and used to assist the development of the EBSDReader and GrainTracker tools in MOOSE. In the following, the reconstruction procedure is briefly introduced. The same procedure was applied to reconstruct the initial and final microstructures of the UO2-S7 sample.

The LANL datasets containing the characterized microstructures were reconstructed by: 1) using MATLAB to read in the raw data, 2) formatting the data so that it is in a usable form, and 3 ), creating a MARMOT compatible output file. This is illustrated in Fig.2, with the file header and first five rows of elemental data output from the UO2-524 dataset. Within the MARMOT/MOOSE framework, two key algorithms (EBSDReader and GrainTracker) were modified to enable phase field simulations using the synchrotron datasets. EBSDReader allows for element based experimental data to be imported and utilized for initialization of the necessary phase field variables. It stores relevant material properties such as crystal orientation, phase, crystal symmetry, and grain index for each element in the datasets. Grain averaged data is also calculated and stored. Both the grain averaged and elemental based data structures are available for later use by any other kernel, material, or auxiliary system within the MARMOT/MOOSE framework.

In Fig.3 the reconstructed UO2-524 microstructure was shown (Fig.3(a)) as an example of the reconstruction. The domain is $582.4 \mathrm{~mm} \times 582.4 \mathrm{~mm} \times 42.7 \mathrm{~mm}$ in size and is composed of 3.6 million elements representing 132 million degrees of freedom. In addition to Allen-Cahn equations for grain growth, split Cahn-Hilliard equations were also used in MARMOT simulations for the porosity, which is conserved during annealing. The simulated annealing was done at $2273 \mathrm{~K}$ for 200 minutes. The 1620 grains were represented by 35 order parameters within GrainTracker. Both adaptive time stepping and adaptive mesh refinement were utilized. The simulation took 51 hours to complete 85 time steps using 144 nodes with a total of 864 MPI processes. Given the large number of grain initially and the 3D nature, the efficiency is pretty satisfactory, demonstrating the MARMOT capability of 3D grain growth simulations using experimental microstructures. At the end of the simulation, the evolved microstructure in Fig.3(b) contains 600 grains. Again, we emphasis that in the simulations the effect of off-stoichiometry on GB mobility was not considered. A direct comparison will be made after a model for off-stoichiometry is ready and after receiving the final microstructure data for the same sample from LANL. 


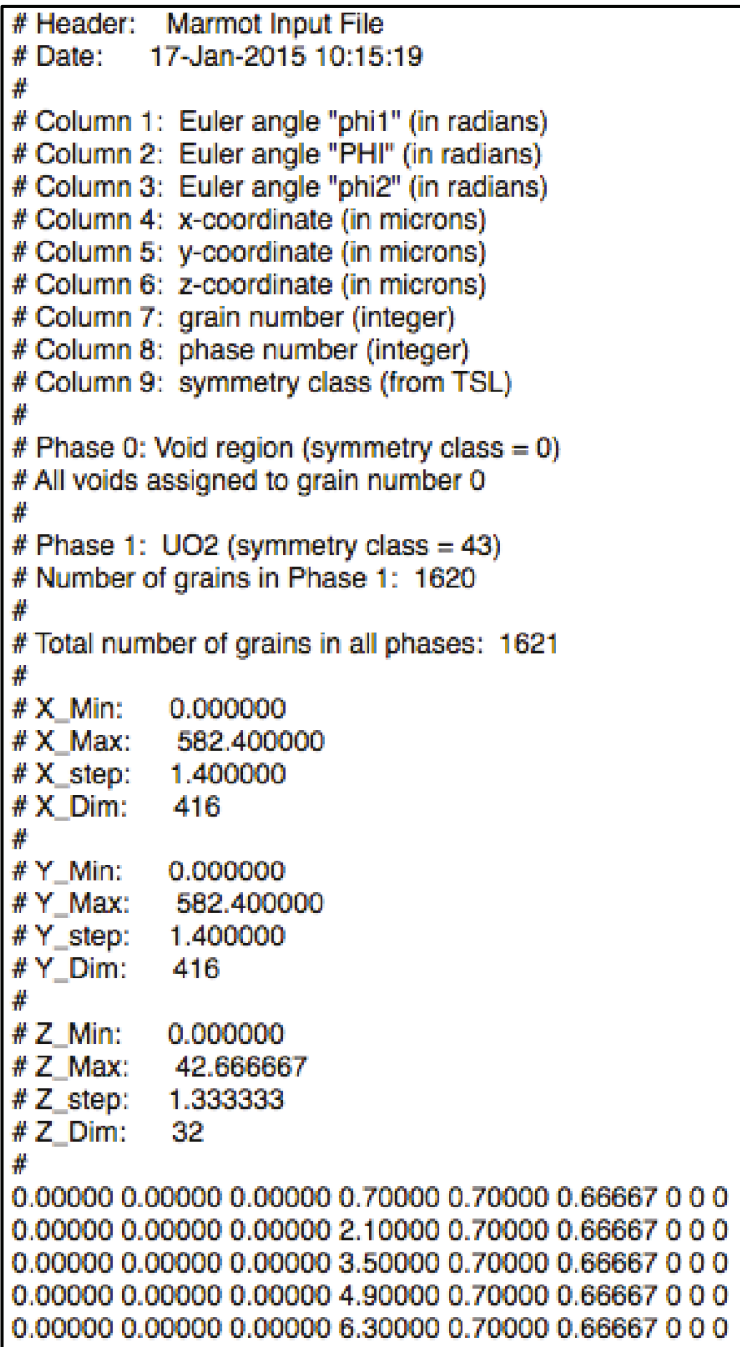

Figure 2: Example of MARMOT input file showing the header information and the first 5 rows of data

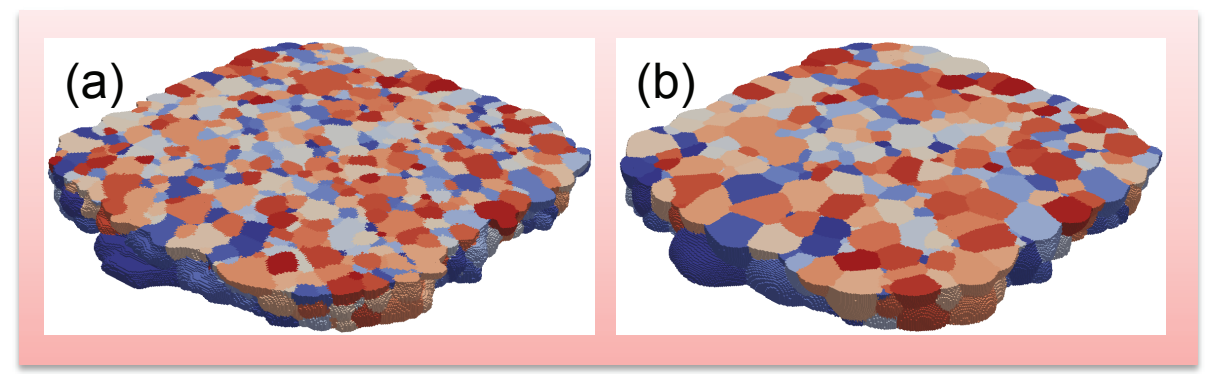

Figure 3: Phase field simulation results of UO2-524 microstructure; (a) reconstructed dataset containing 1620 grains at the beginning of simulation; (b) evolved microstructure after 200 minutes of simulated annealing at $2273 \mathrm{~K}$. The simulation took 85 time steps to reduce the number of grains to 600 . 


\subsection{Comparison of grain growth results from modeling and experiments}

The comparison between modeling and experiments was done using the UO2-S7 sample. This sample is stoichiometric, annealed for 156 minutes at $2273 \mathrm{~K}$. The comparison was made using $2 \mathrm{D}$ simulations since the 3D experimental data was received very recently, and the reconstruction of 3D data was delayed by some inconsistency in the initial and final experimental microstructures. Comparison will be available soon after the reconstruction is finished.

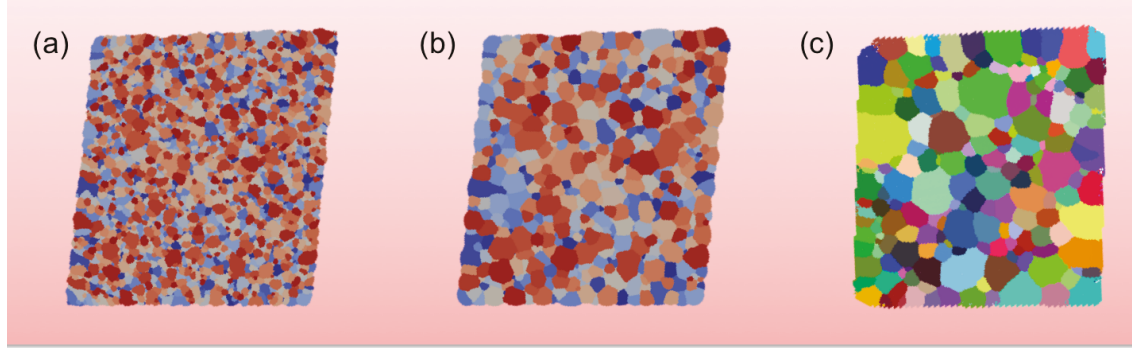

Figure 4: 2D grain growth results for UO2-S7 dataset; (a) initial microstructure with 2091 grains; (b) evolved microstructure contains 502 grains after 156 minutes of simulated annealing at 2273K;

(c) experimental dataset contains 257 grains after 156 minutes of annealing at $2273 \mathrm{~K}$

In Fig.4(a), the reconstructed 2D slice at the center of the UO2-S7 dataset is shown. The same microstructure was used in the MARMOT simulation. In Fig.4(b) and (c) the final microstructure obtained from the simulation and the experiment are shown, respectively. In accordance to the annealing experiments, the simulated annealing lasted for 156 minutes at $2273 \mathrm{~K}$. The parameters used in the simulation are given in Section 2. Again, time and mesh adaptivities were used to enhance the simulation efficiency. As shown in Fig.5, in the simulation the number of grains dropped from 2091 to 502 in 156 minutes, while the final microstructure from experiment contained 257 grains. The rate at which the number of grains dropped decreased with time, showing that the grain growth slowed down as the average grain size increased. As shown in Eq.2 (Section 2), the intrinsic driving force for GB migration is proportional to the inverse grain size. With increasing grain size, the driving force decreases, leading to slower grain growth.

The comparison in 2D represents a good step toward a detailed validation of the MOOSE/ MARMOT framework for grain growth simulations. The results indicate that the grain growth kinetics in the 2D simulation is slower than that in the annealing experiment. This is expected as in $2 \mathrm{D}$ simulations, the curvature and thus the driving force for are underestimated, leading to slower grain growth kinetics. The 2D experimental slice was taken from 3D annealing experiments. It was not affected by the reduced dimensions. This suggests that using a using $2 \mathrm{D}$ simulations to predict $3 \mathrm{D}$ microstructure evolution is tenuous, and a thorough analysis of the $3 \mathrm{D}$ microsturctures is necessary to assess the grain growth model in MARMOT.

The reconstruction of 3D microstructures of the UO2-S7 sample is currently ongoing. After receiving the dataset from LANL, several challenges have been identified during reconstruction. First, the sizes of the two experimental datasets are different. The initial dataset is $108 \mathrm{um}$ in length (27 slices) while the annealed dataset is $200 \mathrm{um}$ (50 slices). Additionally, the position of the sample in the synchrotron was different for the two instances with a substantial rotational difference between them. The different rotational angle effects the Euler angles used to represent the crystallographic texture of the samples. In order to correlate the Euler angles between the two 


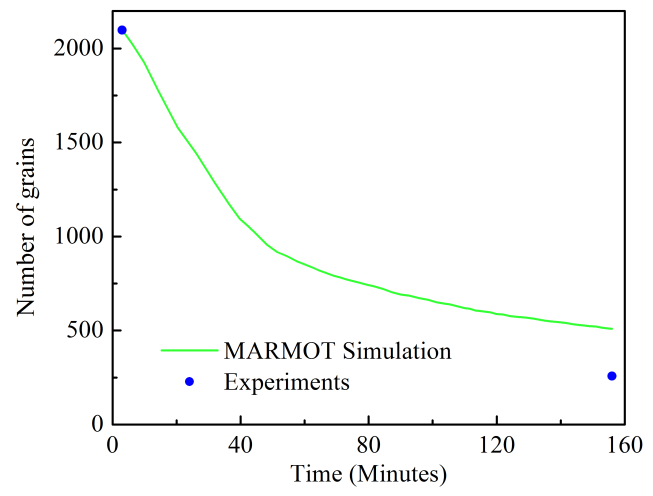

Figure 5: Number of grains in a 2D slice as a function of annleaing time at 2273K.

datasets, a transformation of the angles must be performed to bring them into the same rotational axis. Unfortunately, the exact rotational difference between the two samples is not known, requiring iteration to properly correlate the crystallographic data. Once the crystal data is corrected, we can work to determine the volume within the second dataset that corresponds to the initial dataset and perform the phase field simulations. Conclusions will then be made regarding the efficacy of the MARMOT/MOOSE platform for performing grain growth simulations in $\mathrm{UO}_{2}$. 


\section{Summary}

A rigorous comparison between modeling and experiments requires the modeling to match the experiments regarding initial and experimental conditions. Such an validation approach is demonstrated in Section 3. Experimentally, the initial grain structures of two $\mathrm{UO}_{2}$ samples were characterized using non-destructive x-ray. The thus characterized microstructures were then successfully reconstructed using the MOOSE EBSDReader for MARMOT grain growth simulations. Using the same initial microstructures, the thermal annealing experiments were simulated at the same temperature for the same amount of time. The good matching between the simulations and the experimental conditions allows for valid comparisons.

The EBSDReader and GrainTracker tools in MOOSE were modified to reconstruct the experimental datasets. These tools are shown to be robust for reconstructions. The grain growth simulations were efficient with the help of time and mesh adaptivities in MOOSE. Regarding comparisons between modeling and experimental results, so far only 2D comparison has been finished and $3 \mathrm{D}$ comparison is underway. With $2 \mathrm{D}$ results, it was found that the grain growth kinetics is slower in the simulation than that in the experiment. Such a result is actually expected as in 2D simulations the driving force for grain growth is underestimated in reference to that in 3D. This calls for detailed analysis of the 3D results, which will be available after the inconsistency in the initial and final experimental microstructures is resolved.

The grain growth model in MARMOT is still under active development. In the future, the effects of anisotropic grain boundary energy and mobility, off-stoichiometry and impurity dragging

will be included. A similar validation procedure with well defined experiments will be performed after the new development is finished. 


\section{References}

1. Derek R. Gaston, Cody J. Permann, John W. Peterson, Andrew E. Slaughter, David Andr, Yaqi Wang, Michael P. Short, Danielle M. Perez, Michael R. Tonks, Javier Ortensi, Ling Zou, and Richard C. Martineau. Physics-based multiscale coupling for full core nuclear reactor simulation. Annals of Nuclear Energy, 84:45 - 54, 2015. Multi-Physics Modelling of $\{$ LWR Static and Transient Behaviour.

2. M.R. Tonks, D. Gaston, P.C. Millett, D. Andrs, and P. Talbot. An object-oriented finite element framework for multiphysics phase field simulations. Comp. Mat. Sci., 51(1):20-29, 2012.

3. R.L. Williamson, J.D. Hales, S.R. Novascone, M.R. Tonks, D.R. Gaston, C.J. Permann, D. Andrs, and R.C. Martineau. Multidimensional multiphysics simulation of nuclear fuel behavior. Journal of Nuclear Materials, 423:149 - 163, 2012.

4. M. R. Tonks, D. Schwen, Y. Zhang, P. Chakraborty, X. Bai, B. Fromm, J. Yu, and M. C. Teague. Assessment of marmot: A mesoscale fuel performance code. Technical report, Idaho National Laboratory, 2015.

5. Gunter Gottstein and Lasar S. Shvindlerman. Grain boundary migration in metals: thermodynamics, kinetics, applications, Second Addition. Taylor \& Francis, 2011.

6. Michael R. Tonks, Yongfeng Zhang, Xianming Bai, and Paul C. Millett. Demonstrating the temperature gradient impact on grain growth in $\mathrm{UO}_{2}$ using the phase field method. Materials Research Letters, 2(1):23-28, 2014.

7. N. Moelans, B. Blanpain, and P. Wollants. Quantitative analysis of grain boundary properties in a generalized phase field model for grain growth in anisotropic systems. Phys. Rev. B, 78:024113, 2008.

8. P.V. Nerikar, K. Rudman, T.G. Desai, D. Byler, C. Unal, K.J. McClellan, simon R. Phillpot, Susan B. Sinnott, Pedro Peralta, Blas P. Uberuaga, and Christopher R. Stanek. Grain boundaries in uranium dioxide: Scanning electron microscopy ex- periments and atomistic simulations. Journal of the American Ceramic Society, 94:1893-1900, 2011.

9. JB Ainscough, BW Oldfield, and JO Ware. Isothermal grain growth kinetics in sintered $\mathrm{UO}_{2}$ pellets. J. Nucl. Mater., 49(2):117-128, 1973.

10. MR Tonks, Y Zhang, A Butterfield, and X Bai. Development of a grain boundary pinning model that considers particle size distribution using the phase field method. Modeling and Simulation of Materials Science and Engineering, In press, 2015.

11. Derek Gaston, Cody Permann, David Andrs, John Peterson, and Andrew Slaughter. Moose enhancements towards delivery of an integrated framework. Technical report, Idaho National Laboratory, 2013. 